\title{
Transcatheter arterial chemoembolization alone versus combined with microwave ablation for recurrent small hepatocellular carcinoma after resection: A retrospective comparative study
}

Jie Ji

The First Affiliated Hospital of Nanjing Medical University

Wei Yang

The First Affiliated Hospital of Nanjing Medical University

Hai-Bin Shi

The First Affiliated Hospital of Nanjing Medical University

Sheng Liu

The First Affiliated Hospital of Nanjing Medical University

Wei-Zhong Zhou ( $\triangle$ Xmjbq007@163.com)

The First Affiliated Hospital of Nanjing Medical University

\section{Research Article}

Keywords: recurrence, small hepatocellular carcinoma, microwave ablation, transcatheter arterial chemoembolization

Posted Date: February 11th, 2022

DOI: https://doi.org/10.21203/rs.3.rs-1276386/v1

License: (c) (i) This work is licensed under a Creative Commons Attribution 4.0 International License.

Read Full License 


\section{Abstract}

Purpose: To compare the efficacy and safety of transcatheter arterial chemoembolization combined with microwave ablation (TACE-MWA) versus TACE alone for the treatment of recurrent small hepatocellular carcinoma (sHCC) after resection.

Materials and Methods: From June 2015 to January 2020, a total of 45 patients with recurrent sHCC (size $\leq 3 \mathrm{~cm}$ ) treated by TACE-MWA or TACE were included in this study. The radiological response at 1 month after initial treatment [modified Response Evaluation Criteria in Solid Tumors (mRECIST)], progressionfree survival (PFS), overall survival (OS), and complications were evaluated.

Results: The TACE-MWA group showed a better 1-month complete response (CR) rate (82.3\% vs. 42.8\%, P $=0.013$ ). The corresponding 1-, 3-, and 5-year PFS rates were 76.5\%, 70.6\%, and 70.6\% for the TACE-MWA group, and $56.1 \%, 15.0 \%$, and $15.0 \%$ for the TACE group $(P=0.003)$. The $1-, 3-$, and 5 -year OS rates were $100.0 \%, 82.1 \%$, and $61.5 \%$ for the TACE-MWA group, and $89.0 \%, 58.1 \%$, and $50.8 \%$ for the TACE group (P $=0.389$ ), respectively. Moreover, no major complications related to treatment were observed in either of the groups. Compared with the TACE group, the TACE-MWA group had a significantly lower number of reTACE sessions $(P=0.003)$.

Conclusions: Although TACE alone provides equivalent effectiveness for recurrent sHCC in terms of OS rates, TACE-MWA leads to a better tumor response at 1 month and may prolong tumor PFS time.

\section{Introduction}

Hepatic resection has been established as a curative treatment for hepatocellular carcinoma (HCC) [1-4]. However, the long-term prognosis of hepatic resection has been disappointing because of the high recurrence rates in the remnant liver [5-8]. Previous studies have reported that the cumulative 5-year recurrence rates after hepatic resection are as high as 78\%-96\% [9-11]. Due to the close postoperative follow-up, as well as improved imaging technology, more recurrent small HCC (defined as $\leq 3 \mathrm{~cm}$ ) are diagnosed at an early stage [12]. Therefore, the management of optimal treatment for recurrent sHCC is urgently required.

Repeat hepatectomy is an effective treatment for sHCC recurrence, however, it is limited by the patient's general condition, remnant liver volume, tumor distribution, liver function, and tumor's invasiveness. Miniinvasive therapies, such as radiofrequency ablation (RFA), microwave ablation (MWA) and TACE, have emerged as alternative treatments $[5,7,10,12]$. The combination of TACE and ablation may have several theoretical advantages over either monotherapy [13-15]. First, the decreased blood flow induced by TACE reduces heat loss, thereby increasing the size of the ablation ablative zone. In addition, the lipiodol of TACE deposited in the tumor can be a mark for the ablation needle insertion and facilitates evaluation of the ablative margin[16]. TACE combined with RFA for sHCC has been shown to be superior to TACE alone [14]. Compared with RFA, MWA has less heat-sink effect and could achieve a larger coagulation volume 
in a shorter procedural time [17]. However, given limited literature, it remains unknown that whether TACEMWA is effective in the treatment of recurrent sHCC.

The purpose of this study was to compare TACE-MWA with TACE alone for recurrent sHCC. We compared tumor response at 1 month, and progression-free survival (PFS), overall survival (OS), and complications after treatment with TACE-MWA or TACE alone.

\section{Materials And Methods}

\section{Patient selection}

The study complied with the Declaration of Helsinki on human research and its later amendments and obtained approval of our institutional review board. Due to the retrospective study design, written informed consent was waived (the Ethics Committee of the First Affiliated Hospital with Nanjing Medical University). A total of 45 patients with recurrent sHCC treated with conventional TACE or TACE -MWA, were enrolled in our center from June 2015 to January 2020 (Fig. 1). The diagnosis of HCC was confirmed by pathology or imaging in accordance with the European Association for the Study of the Liver criteria guidelines. The inclusion criteria were as follows: (a) first intrahepatic recurrent HCC after initial resection, (b) recurrent three or fewer tumors under $3 \mathrm{~cm}$ prior to receiving TACE or TACE-MWA, (c) Child-Pugh A or B, (d) absence of extrahepatic metastasis or macrovascular invasion, and (e) Eastern Cooperative Oncology Group performance score $\leq 2$. The exclusion criteria included: (a) additional treatments other than TACE or MWA, (b) secondary malignancies, (c) follow-up period less than 1 year or incomplete medical record.

\section{TACE}

A $5 \mathrm{~F}$ catheter was introduced through the femoral artery, to assess liver vascular anatomy and to confirm the patency of the portal vein by visceral angiography. Then, a selected micro-catheter (Terumo, Tokyo, Japan) was then super-selectively inserted into the hepatic lobe or hepatic segmental artery branch. A 1:1 mixed suspension of iodized oil (1-10 mL, Andre' Guerbet Laboratories,) and epirubicin (20-40 mg, Pharmorubicin, Pfizer, Wuxi, China) were infused into the artery through the catheter, depending on the size and number of the tumor. Finally, the embolization was performed using a gelatin sponge until the blood supply of the tumor significantly decreased.

\section{TACE-MWA}

After TACE procedures, MWA was applied sequentially within 2 weeks. MWA was performed using a microwave ablation system (ECO Corporation, Nanjing, China) at $2450 \pm 50 \mathrm{MHz}$ with continuous adjustable power output of 0-100 W. A plain scan of the tumor location was obtained by CT, to determine the optimal puncture trajectory. Under general anesthesia, the electrode probe was inserted to reach the opposite edge of the tumor through its center. The correct puncture position was ensured after an additional scan. Then, the microwave power and duration adjusted based on the tumor's location and 
size, and the coagulation range was required to be $5-10 \mathrm{~mm}$ beyond the edge of the tumor. After the ablation, an addition CT scan was performed to verify the coagulation zone.

\section{Follow-up protocol}

One month after the TACE-MWA or TACE procedure, each patient was followed by serum tumor makers, as well as enhanced liver magnetic resonance imaging (MRI) or CT. If no viable tumor was indicated, the follow-up intervals were extended to 2-3 months thereafter. If residual viable lesions were detected in the scan, repeated TACE was performed in the TACE group. Depending on tumor shape, size, or number, repeated TACE or MWA was performed in the TACE-MWA group. Based on mRECIST, we assessed PFS, which was defined as the interval from the first TACE treatment to tumor progression, death, or last follow-up. OS was defined as the interval from the first TACE treatment to death or last follow-up.

\section{Statistical analysis}

The baseline characteristics of the patients were expressed as mean \pm standard deviation (SD), median and interquartile range (IQR) or percentage. Comparisons between the two groups were done using the Student's $t$ test or Mann-Whitney Utest for continuous data and the chi-square test for categorical data. The PFS and OS were calculated by the Kaplan-Meier method and the groups were compared by logrank test. All of the statistical tests were two-sided, and a difference was considered significant when $P$ was lower than 0.05. The data were analyzed using SPSS (version 22.0, IBM Corp, Armonk, NY, USA) and $\mathrm{R}$ version 3.3.2 (R Foundation for Statistical Computing, Vienna, Austria).

\section{Results}

\section{Patients' characteristics}

Clinical and histopathological data are summarized (Table 1). In this study, 15 patients (33.3\%) had died and 30 patients $(66.7 \%)$ were still alive. The median follow-up time for the cohort was 44.1 months. The single largest tumor size ranged from 0.8 to $3.0 \mathrm{~cm}$. With respect to the number of tumors, 29 patients (64.4\%) had 1 tumor, 12 patients $(26.6 \%)$ had 2 tumors, and 4 patients $(9.0 \%)$ had 3 tumors. In the TACEMWA group the largest single tumor size ranged from 1.0 to $3.0 \mathrm{~cm}$. After the initial combined treatment, there were 0 to 9 re-TACE sessions per patient, with a mean of 1.4 sessions. In the TACE alone group, the single largest tumor size ranged from 0.8 to $3.0 \mathrm{~cm}$. After the initial TACE procedure, there were 0 to 7 re-TACE sessions per patient, with a mean of 2.8 sessions.

\section{Radiological treatment response}

Radiological response was assessed based on mRECIST criteria at 1 month after treatment [Table 2]. Evidently, the TACE-MWA group showed higher proportions of patients with complete response (CR) $[(82.3 \%$ vs. $42.8 \%), P=0.013]$ and objective response (sum of $C R$ and partial response) $[(100.0 \%$ vs 71.4\%), $P=0.017$ ] than the TACE group. 
In the entire cohort, the median PFS was 16.8 months. There were 28 patients (62.2\%) within the entire cohort experiencing tumor progression. In the TACE alone group, the median PFS was 14.1 months, with 23 patients $(82.1 \%)$ having progression. In the TACE+MWA group, 5 patients $(29.4 \%)$ had signs of progression at the time of analysis. The median PFS was not applicable because more than $50 \%$ of the tumor lesions were still stable until the time of the last follow-up. There was a significant difference in PFS between the TACE group and the TACE+MWA group $(P=0.003$, Fig. $2 A)$.

\section{OS}

At the time of analysis, 15 patients (33.3\%) had died and 30 (66.7\%) were still alive. The 1-, 3-, and 5-year OS rates for the cohort were $93.2 \%, 64.5 \%$, and $55.0 \%$, respectively. In the TACE group, 12 patients (42.8\%) had died at the time of analysis, and 1-, 3-, and 5-year OS rates were $89.0 \%, 58.1 \%$, and $50.8 \%$, respectively. In the TACE+MWA group, three patients (17.6\%) had died at the time of analysis, and 1-, 3-, and 5 -year OS rates were $100.0 \%, 82.1 \%$, and $61.5 \%$, respectively. There existed no statistical difference in OS rates between the groups (Fig. 2B).

\section{Safety evaluation}

Based on the Score Index for Radiosurgery (SIR) grading system, several adverse events were noted and graded. Post-procedure minor complications (vomiting, fever, nausea and abdominal pain) occurred in $23.5 \%(4 / 17)$ of the patients in the TACE-MWA group and in $42.8 \%(12 / 28)$ in the TACE group. During the MWA procedure, most patients complained of local burning pain, but it was generally tolerable after use of basal anesthesia. After the procedure, some patients reported having fever and local pain, but these were not severe. There was no significant difference in the incidence of complications between the two groups $(P=0.219)$. All of these adverse effects were transient and were relieved after symptomatic treatment. No major complications (organ injury, hemorrhage, ascites, and liver failure) and death related to treatment were observed for either of the procedures.

\section{Discussion}

In our retrospective analysis of 45 patients with recurrent small HCC, the 5-year PFS of patients receiving the combination therapy (37.5\%) was higher than that of patients receiving TACE alone $(18.7 \%, \mathrm{P}=$ 0.003 ), indicating that the combination therapy showed better tumor response and may prolong tumor PFS time.

The combination treatment of TACE and ablation did not show better survival outcomes than ablation monotherapy in primary small HCC (Chai et al. 2021) [18], while the results remain vague in recurrent small HCC after resection. Our study demonstrated that TACE-MWA compared with TACE alone could offer better tumor control for patients with recurrent small HCC. Similar to the results from the study by Chen et al. [19], our study showed that $82.7 \%$ tumors were completely ablated in the TACE-MWA group , 
higher than $42.8 \%$ tumors in the TACE alone group $(P=0.013)$. Moreover, objective response rate $(C R+P R)$ was also significantly higher for the TACE-MWA group than the TACE group $(100 \%$ vs. $71.4 \%, P=0.017)$. Significant difference was found in PFS between the TACE and the TACE-MWA groups $(P=0.003)$,

The reason why TACE-MWA achieved better tumor control than TACE alone may lie in the synergistic effect of these two treatments. First, TACE could reduce the heat-sink effect of ablation by obstructing the tumor-feeding artery, thus in turn enhancing the coagulation effect of MWA. Second, chemotherapy agents may cause a heat-sensitizing effect and thermal injury may sensitize tumors to the chemotherapeutic agents [16]. Third, lipiodol was deposited in the tumor and peri-tumor margin after TACE, which could help to accurately assess the tumor size and facilitated the subsequent ablation. In addition, TACE also could help to control these micro-lesions, reducing the recurrence of tumor.

The cumulative OS rates at 5 years were $61.1 \%$ for TACE-MWA and $50.3 \%$ for TACE alone in recurrent sHCC patients, which is similar to previously reported 5-year cumulative OS rates of TACE-RFA (46\%$60 \%)[5,14]$. Although we found no significant difference in OS between the TACE group and the TACEMWA group, complete necrosis after TACE has been linked to good survival outcome in patients with recurrent HCC [20]. A tendency favoring TACE-MWA was also found in our study. The findings of this study suggest that TACE could be used as an alternative treatment option for patients with small recurrence who are ineligible for curative therapies. Thus, our study may facilitate the development of a treatment algorithm for recurrent HCC.

There were some limitations in our study. First, the principal limitation was its susceptibility to selection bias owing to its retrospective nature. In the TACE group, most of the procedures were performed early in this study period, while most in the TACE-MWA group were performed later in this study period. Therefore, the influence of measured and unmeasured confounders on the outcome of patients was inevitable. Second, given the relatively small number of patients enrolled in this study, a larger number of subjects and a prospective study design are desirable to conform the results.

\section{Conclusion}

In conclusion, TACE and TACE-MWA may lead to a similar OS in patients with recurrent small HCC after resection. However, TACE-MWA shows a better tumor response at 1 month and may prolong tumor PFS time.

\section{Abbreviations}

TACE: transcatheter arterial chemoembolization, MWA: microwave ablation, HCC: hepatocellular carcinoma, PFS: progression-free survival, OS: overall survival, CR:complete response, MRI: magnetic resonance imaging, SD: standard deviation, IQR:interquartile range.

\section{Declarations}




\section{Authors' contributions}

Concepts/study design: W-ZZ, H-BS, LS, data analysis/ interpretation: JJ, YW, data collection: JJ,, manuscript drafting: JJ, statistical analysis: JJ, All authors read and approved the final manuscript.

\section{Funding}

None

\section{Availability of data and materials}

The datasets generated and/or analysed during the current study are not publicly available囚because it is related to subsequent research $\bigotimes$ but are available from the corresponding author upon reasonable request.

\section{Ethics approval and consent to participate}

Study protocol followed the guidelines of the World Medical Association Declaration of Helsinki and were approved by the Ethics Committee of the First Affiliated Hospital with Nanjing Medical University. Due to the retrospective study design, written informed consent was waived (the Ethics Committee of the First Affiliated Hospital with Nanjing Medical University).

\section{Consent for publication}

Not applicable.

\section{Acknowledgements}

Not applicable.

\section{Competing interests}

The authors declare that they have no competing interests.

\section{References}

1. Bray, F., Ferlay, J., Soerjomataram, I., Siegel, R.L., Torre, L.A., Jemal, A. Global cancer statistics 2018: Globocan estimates of incidence and mortality worldwide for 36 cancers in 185 countries. CA Cancer J. Clin. 2018, 68, 394-424.

2. Yang, J.D.,Roberts, L.R. Hepatocellular carcinoma: A global view. Nat. Rev. Gastroenterol. Hepatol. 2010, 7, 448-458.

3. Rahbari NN, Mehrabi A, Mollberg NM, et al. Hepatocellular carcinoma: current management and perspectives for the future. Ann Surg 2011, 253:453-4694. 
4. Fuks D, Dokmak S, Paradis V, Diouf M, Durand F, Belghiti J. Benefit of initial resection of hepatocellular carcinoma followed by transplantation in case of recurrence: an intention-to-treat analysis. Hepatology 2012, 55:132-1405.

5. Peng ZW, Zhang YJ, Liang HH, Lin XJ, Guo RP, Chen MS. Recurrent hepatocellular carcinoma treated with sequential transcatheter arterial chemoembolization and RF ablation versus RF ablation alone: a prospective randomized trial. Radiology 2012, 262:689-700

6. Ercolani G, Grazi GL, Ravaioli M, et al. Liver resection for hepatocellular carcinoma on cirrhosis: univariate and multivariate analysis of risk factors for intrahepatic recurrence. Ann Surg 2003, 237:536-543

7. Eguchi S, Matsumoto S, Hamasaki K, et al. Reevaluation of lipiodolized transarterial chemoembolization therapy for intrahepatic recurrence of hepatocellular carcinoma after curative liver resection. J Hepatobiliary Pancreat Surg 2008, 15:627-633

8. Kawano Y, Sasaki A, Kai S, et al. Prognosis of patients with intrahepatic recurrence after hepatic resection for hepatocellular carcinoma: a retrospective study. Eur J Surg Oncol 2009, 35:174-179

9. Fuks D, Dokmak S, Paradis V, Diouf M, Durand F, Belghiti J. Benefit of initial resection of hepatocellular carcinoma followed by transplantation in case of recurrence: an intention-to-treat analysis. Hepatology 2012, 55:132-140

10. Ho CM, Lee PH, Shau WY, Ho MC, Wu YM, Hu RH. Survival in patients with recurrent hepatocellular carcinoma after primary hepatectomy: comparative effectiveness of treatment modalities. Surgery 2012, 151:700-709

11. Berretta $M$, Cavaliere $C$, Alessandrini $L$, et al. Serum and tissue marks in hepatocellular carcinoma and cholangiocarcinoma: clinical and prognostic implications. Oncotarget. 2017, 8: 14192-14220.

12. Chan AC, Poon RT, Cheung TT, et al. Survival analysis of re-resection versus radiofrequency ablation for intrahepatic recurrence after hepatectomy for hepatocellular carcinoma. World J Surg 2012 , 36:151-1

13. Chen Rongxin, Gan Yuhong, Ge Ninglin et al. Transarterial Chemoembolization versus Radiofrequency Ablation for Recurrent Hepatocellular Carcinoma after Resection within Barcelona Clinic Liver Cancer Stage 0/A: A Retrospective Comparative Study.[J] .J Vasc Interv Radiol, 2016, 27 : 1829-1836.

14. Kim W, Cho SK, Shin SW, Hyun D, Lee MW, Rhim H. Combination therapy of transarterial chemoembolization (TACE) and radiofrequency ablation (RFA) for small hepatocellular carcinoma: comparison with TACE or RFA monotherapy. Abdom Radiol (NY). 2019,44(6):2283-2292

15. Sun Y, Ji S, Ji H, Liu L, Li C. Clinical efficacy analysis of transcatheter arterial chemoembolization (TACE) combined with radiofrequency ablation (RFA) in primary liver cancer and recurrent liver cancer. J BUON. 2019,24(4):1402-1407.

16. Rossi S, Garbagnati F, Lencioni R, et al. Percutaneous radio-frequency thermal ablation of nonresectable hepatocellular carcinoma after occlusion of tumor blood supply. Radiology. 2000,217(1):119-126 
17. Poulou LS, Botsa E, Thanou I, Ziakas PD, Thanos L. Percutaneous microwave ablation vs radiofrequency ablation in the treatment of hepatocellular carcinoma. World J Hepatol. 2015,7(8):1054-1063.

18. Chai NX, Chapiro J, Petukhova A, et al. Thermal ablation alone vs thermal ablation combined with transarterial chemoembolization for patients with small $(<3 \mathrm{~cm})$ hepatocellular carcinoma [published online ahead of print, 2021 Feb 11]. Clin Imaging. 2021,76:123-129.

19. Chen QF, Jia ZY, Yang ZQ, Fan WL, Shi HB. Transarterial Chemoembolization Monotherapy Versus Combined Transarterial Chemoembolization-Microwave Ablation Therapy for Hepatocellular Carcinoma Tumors $\leq 5 \mathrm{~cm}$ : A Propensity Analysis at a Single Center.Cardiovasc Intervent Radiol. 2017,40(11):1748-1755.

20. Shim JH, Kim KM, Lee YJ, et al. Complete necrosis after transarterial chemoembolization could predict prolonged survival in patients with recurrent intrahepatic hepatocellular carcinoma after curative resection. Ann Surg Oncol. 2010,17(3):869-877.

\section{Tables}

Table 1. Baseline characteristics of patients 


\begin{tabular}{|c|c|c|c|}
\hline Variables & $\begin{array}{l}\text { TACE } \\
(n=28)\end{array}$ & $\begin{array}{l}\text { TACE - MWA } \\
(n=17)\end{array}$ & $P$ value \\
\hline \multicolumn{4}{|l|}{ Sex, n (\%) } \\
\hline Male & 24 & 15 & 1.000 \\
\hline Female & 4 & 2 & \\
\hline Age $\llbracket$ years $\rrbracket$ & $58.25 \pm 10.15$ & $60.29 \pm 9.90$ & 0.512 \\
\hline \multicolumn{4}{|l|}{ Initial HCC resection data } \\
\hline Size of resected HCC, median (IQR), cm & $3.5(2.1-5.0)$ & $3.0(1.7-5.5)$ & 0.624 \\
\hline No. of resected HCCs, median (IQR), n & $1.0(1.0-1.0)$ & $1.0(1.0-1.5)$ & 0.502 \\
\hline \multicolumn{4}{|l|}{ Edmonson grade, $\mathrm{n}(\%)$} \\
\hline 1 & 12 & 8 & 0.487 \\
\hline II-III & 15 & 7 & \\
\hline III & 1 & 2 & \\
\hline \multicolumn{4}{|l|}{ BCLC stage邓n (\%) } \\
\hline A & 25 & 16 & 0.990 \\
\hline B & 3 & 1 & \\
\hline \multicolumn{4}{|l|}{ Recurrence stage data } \\
\hline Time to recurrence, median (IQR), days & $319 \llbracket 31-1478 \rrbracket$ & $136 \rrbracket 47-1872 \rrbracket$ & 0.012 \\
\hline Largest tumor size, median (IQR), cm & $1.6(1.3-2.6)$ & $1.4(1.1-2.0)$ & 0.254 \\
\hline Tumor number, median (IQR), n & $1.0(1.0-2.0)$ & $1.0(1.0-2.0)$ & 0.237 \\
\hline \multicolumn{4}{|l|}{ HBV, n (\%) } \\
\hline Yes & 28 & 17 & NA \\
\hline No & 0 & 0 & \\
\hline \multicolumn{4}{|l|}{ AFP, n (\%) } \\
\hline$\geq 400 \mathrm{ng} / \mathrm{mL}$ & 5 & 2 & 0.902 \\
\hline$\varangle 400 \mathrm{ng} / \mathrm{mL}$ & 23 & 15 & \\
\hline PLT, median (IQR), $10^{\wedge} 9 / \mathrm{L}$ & $113.0(74.7-154.5)$ & $103.0(84.0-159.0)$ & 0.858 \\
\hline ALT, median (IQR) , IU/L & $25.1(19.8-31.3)$ & $20.5(15.5-25.5)$ & 0.046 \\
\hline AST, median (IQR), IU/L & $28.5(21.3-40.9)$ & $22.9(21.0-28.2)$ & 0.050 \\
\hline
\end{tabular}




\begin{tabular}{|c|c|c|c|}
\hline ALB, median (IQR), g/L & $39.0(37.9-42.9)$ & $40.0(36.5-44.6)$ & 0.835 \\
\hline TBIL, median (IQR), $\mu \mathrm{mol} / \mathrm{L}$ & $15.3(11.9-18.8)$ & $14.7(12.7-18.3)$ & 0.891 \\
\hline PT, median (IQR), s & $12.6(12.1-13.2)$ & $12.5(12.0-13.1)$ & 0.429 \\
\hline INR, median (IQR) & $1.1(1.0-1.2)$ & $1.1(1.0-1.1)$ & 0.313 \\
\hline \multicolumn{4}{|l|}{ Child-Pugh, n (\%) } \\
\hline$A$ & 26 & 17 & 0.703 \\
\hline B & 2 & 0 & \\
\hline \multicolumn{4}{|c|}{$\begin{array}{l}\text { Abbreviations: IQR, interquartile range; HBV, hepatitis B virus; AFP, a-fetoprotein; PLT, platelet; } \\
\text { PT:prothrombin time; INR, international normalized ratio; ALT, alanineaminotransferase; AST, } \\
\text { aspartate aminotransferase; ALB, albumin; TBIL, totbilirubin; NA, not application; TACE, transarterial } \\
\text { chemoembolization; MWA: microwave ablation; BCLC, barcelona clinic liver cancer. }\end{array}$} \\
\hline
\end{tabular}

Table 2. Tumor response at 1 month between the two groups

\begin{tabular}{|lccc|}
\hline Radiological response & TACE $(\mathrm{n}=28)$ & TACE-MWA $(\mathrm{n}=17)$ & P value \\
\hline Complete response & 12 & 14 & 0.005 \\
\hline Partial response & 8 & 3 & \\
Stable disease & 4 & 0 & \\
Progressive disease & 4 & 0 & \\
Abbreviations: TACE, transarterial chemoembolization; MWA: microwave ablation. \\
\hline
\end{tabular}

\section{Figures}




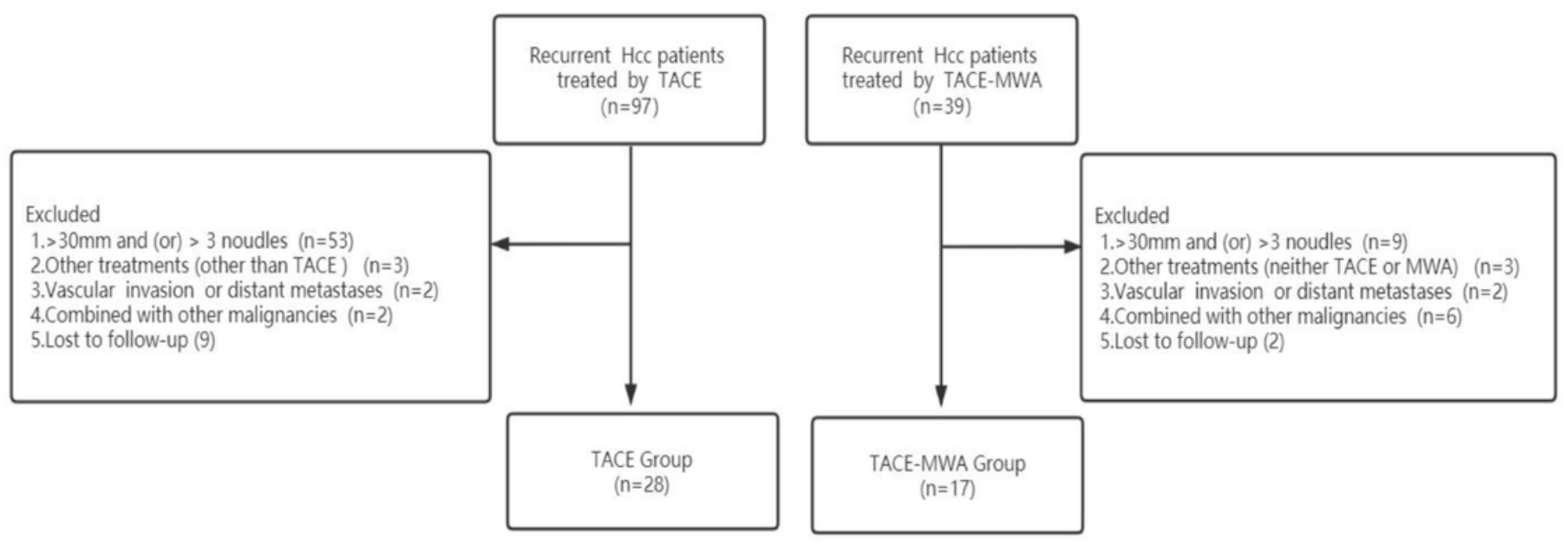

Figure 1

Flow chart of the study population

Figure 2

Progression-free survival (PFS) and overall survival (OS) curves with risk tables for patients with recurrent small HCC after hepatectomy who underwent TACE-MWA or TACE alone. A. PFS curves. B. OS curves. 